\title{
Selective Light and Activity of Antioxidant Enzymes in Case of Infection of Wheat Sprouts with Fusarium Oxysporum \\ ${ }^{* 1}$ T.P. Yakushenkova, ${ }^{2}$ V.B. Michailova \\ 1,2 Kazan Federal University \\ Email: tyakushekfu@gmail.com, Contact: 8951060129
}

\author{
Received: $21^{\text {st }}$ October 2017 Accepted: 16 $^{\text {th }}$ November 2017, Published: $31^{\text {st }}$ December 2017
}

\begin{abstract}
The effect of blue and red light on the growth and activity of antioxidant enzymes in winter wheat sprouts, in case of infection with Fusarium oxysporum was investigated. It was shown, that blue and red spectral regions could regulate the activity of antioxidant enzymes in nine-day wheat germs, both in normal state and when infested with Fusarium oxysporum. Red spectral region increased the activity of peroxidase, catalase, ascorbate peroxidase, under the influence of Fusarium oxysporum, while only the peroxidase activity was increased in blue light. When colonizing the roots of sprouts by Fusarium oxysporum, lipid peroxygenation decreases in red and blue light, which may indicate their resistance to the studied phytopathogen. It was established, that the red spectral region stimulated the growth parameters of the aboveground and underground parts of wheat sprouts. In case of germs infestation with the pathogenic fungus Fusarium oxysporum, significant production of dry weight by the underground partsof the plants was observed.
\end{abstract}

Keywords: Wheat Sprouts, Blue and Red Light, Activity of Antioxidant Enzymes, Fusarium oxysporum, Malonic dialdehyde Content, Dry Weight.

\section{Introduction}

Among the priority areas of modern crop farming are the researches in the field of plant resistance to pathogenic organisms. The knowledge of the mechanisms, using which plants develop systemic stability, is of great practical importance, since annually the various pathogens of diseases of cultivated plants deteriorate the commercial qualities of food products, and reduce the total yield by $40 \%$ [Hammond-Kosack et al., 2000]. Fusarium oxysporum plays the role of a quiet killer - as far as pathogenic histamines of this fungus can keep in the soil and on plant residues for 30 years, and remain virulent and capable of infecting plants. Conidia of this fungus are transferred by water, insects, through instruments and by air. Mass development of fungi is observed in the conditions of high humidity and temperature.

Roots browning and rot, thinning of the root part of the stem are observed in the affected plants. Under the influence of $F$. oxysporum, plants faded and, as a rule, then they died. Fusarium affects all types of agricultural plants [Michielse, 2009]. In cereals, in addition to rotting the roots, stems often die off, or ears without grain are observed. Plants, affected by rot, are easily pulled from the soil. The disease is found everywhere [Leslie, 2006].

At present, agricultural ecosystems consist of genetically impoverished populations of agricultural plants. They grow at high density, at large spatial scales, herewith the regional species composition of agricultural crops vary only slightly from year to year [Read, 2016].

Despite the significant progress, achieved over the past two decades in the manipulation of genes, the fully resistance of plants to biotic stresses has not been created up to now. Light is one of the most important external factors, having a direct impact on plant development [Casal, 2013; Xinyu Zhang et al., 2017]. The specific effect of light of different spectrum on the growth, development and formation of resistance to pathogenic organisms was studied and proved [Cheng et al., 2004]. It was shown, that the invasiveness and virulence of many pathogens depended on the spectral quality of light [Ricci, 2015; Schuerger, 1997]. At present, the mechanisms of the effect of different spectral composition of light on the infected plant organism are still underexplored. In this regard, the following goal of the research was set: to study the activity of antioxidant protective system of wheat germs, grown in the conditions of red and blue light, under the influence of the pathogenic fungus $F$. oxysporum.

\section{Methods}

As the object of the study, we selected winter wheat of the variety Kazan 560 (Triticum aestivum L.), which has been grown for nine to fourteen days in special germinating box, divided into three lightinsulated blocks: 1 - white light (WL), the light source - luminescent lamps LDS- 40, 2 - blue light (BL), the light source - luminescent lamps LH-40, transmission peak - 420-460 nm and 3 - red light (RL), the light source - luminescent lamps LK-40, transmission peak - 620-640 nm for 12-hour photoperiod. Wheat germs were obtained by growing in cuvettes with sterile river sand on distilled water (control variant) and adding Fusarium oxysporum pathogenic fungi to the growth medium at a concentration of $1 \times 10^{-6}$ colonies $/ \mathrm{ml}$ (test variant).

Determination of the activity of soluble peroxidase in homogenate 
The activity of the enzyme Prxs was determined according to the method of Boyarkin [Ermakov et al., 1987] in homogenate. This method is based on the defining the oxidation reaction rate of benzidine to the formation of blue product of its oxidation, in the presence of $\mathrm{H}_{2} \mathrm{O}_{2}$ and Prxs. The investigations were made using the spectrophotometer Unico; then the fall of optical density was measured at $590 \mathrm{~nm}$ during $120 \mathrm{~s}$.

Determination of catalase activity in homogenate The spectrophotometric method, proposed by Aeby [Aeby, 1984] was used for determining the activity of CAT (EC 1.11.1.6). This method is based on defining the decomposition rate of $\mathrm{H}_{2} \mathrm{O}_{2}$ by catalase of the test sample, to form water and oxygen.

Determination of the activity of ascorbate peroxidase APX activity (EC 1.11.1.7) was determined according to the method of Verma and Dubey [Verma, Dubey, 2003]. This method is based on determining the rate of decomposition of $\mathrm{H}_{2} \mathrm{O}_{2}$ with ascorbate peroxidase of the test sample, to form water and dehydroascorbate.

Determination of the level of lipid peroxidation (content of malonic dialdehyde)
The level of lipid peroxidation (LPO) is judged by the accumulation of the LPO product malondialdehyde. The MDA content is estimated by the degree of accumulation of the product of its reaction with thiobarbituric acid [Kumar, Knowles, 1993].

The control of test plants for $F$. oxysporum infestation was carried out on the 14th day of their cultivation. Tested wheat sprouts had dark brown stains on the stems.

\section{Results and Discussion}

The influence of spectral composition of light on the morphological characteristics of wheat sprouts

The processes of plants are in close relationships both with the intensity of light and with its spectral composition. It was shown, that different spectra of light regulated the growth, development, productivity and photosynthetic processes of plants, as in vitro, as in vivo.

When studying the effect of qualitative composition of light on the morphological parameters of the above-ground length (Figure 1) and the underground part (Figure 2) of wheat sprouts, we showed that infestation with pathogenic fungi, as a whole, negatively affected the growth parameters, especially the length of the foot end of the plant.

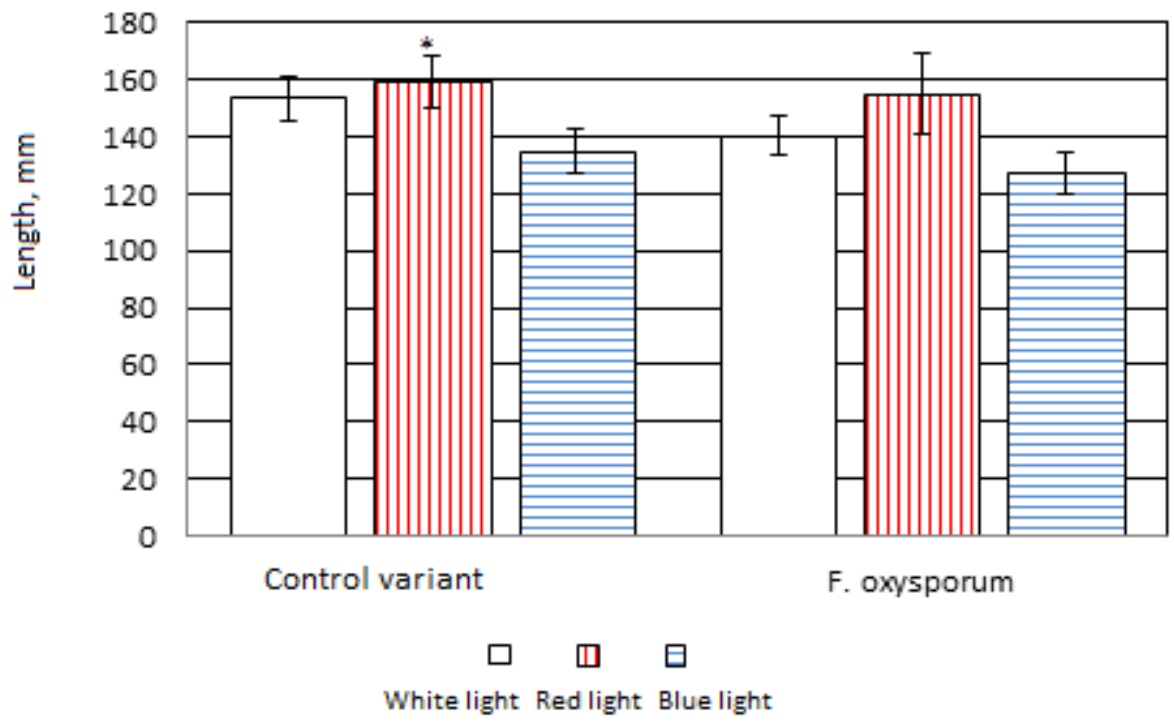

Figure 1 - The influence of the spectral composition of light on the length of the above ground part of 9day-old sprouts of wheat of the variety Kazan 560, in control variant and in case of their infestation with $F$. oxysporum (* indicates the adequacy of this parameter, compared with the WL parameter). 


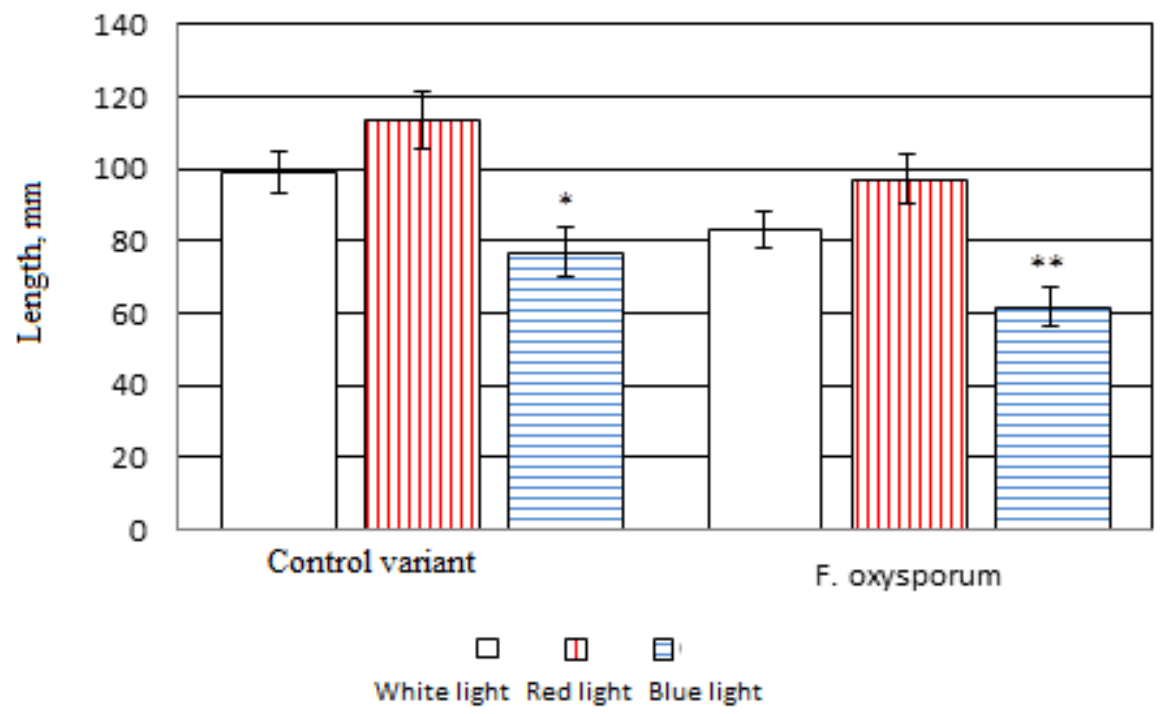

Figure 2 - The influence of spectral composition of light on the length of the underground part of 9-day-old sprouts of wheat of the variety Kazan 570, in the control variant and in case of their infestation with F. oxysporum (* indicates the adequacy of this parameter, compared with the WL parameter, ** indicates the adequacy of this parameter, compared with the RL parameter).

Fungi of the genus Fusarium affect various parts of many plants. Most fungi of this genus are phytotrophs [Michielse, 2009; Leslie, 2006; Alabouvette, 2009]. F. oxysporum fungus is the causative agent of tracheomycosis wilt, which manifests during the period of germination. A decrease in growth and development is observed when the plants are infested [Tsukagoshi, 2010].

The inhibition of length of the aboveground and underground parts of plants, infested with pathogenic fungusF.oxysporum, was defined by us. The decrease of growth parameters under the influence of fungi hypha occurs as a result of occlusion of xylem vessels, that's lows down the transport of nutrients to the upper parts of the plant.

Under the influence of light of different composition and $F$. oxysporum on wheat germs, we recorded a positive effect of RL on the growth of healthy and infested plants, in comparison with samples, grown in BL and WL. Blue light, on the contrary, had an inhibitory effect on the development of plants, in comparison with RL and WL. At the same time, it was previously shown, that the red portion of the spectrum causedthe lignification of the cell wall [Yakushenkova et al., 2012], preventing the penetration of the phytopathogen into the root system and its spread along the xylem.

Dry weight production by the sprouts of wheat The dry weight production is the most important integral indicator, characterizing the growth of both healthy and infested plants, the growth rate of which depends on growth conditions and growth phases.

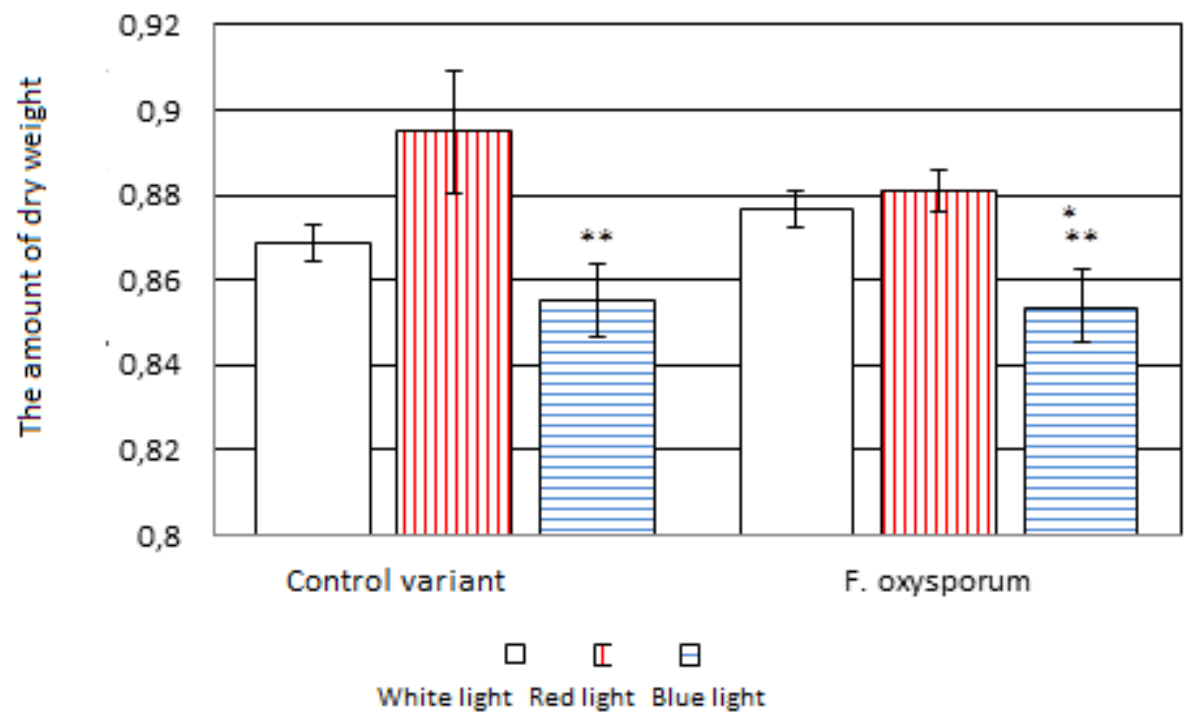


Figure 3 -The influence of light of different spectral composition on the dry weight content of the wheat roots of the variety Kazan 560, in the control variant and in case of their infestation with $F$. oxysporum (* indicates the adequacy of this parameter, compared with the WL parameter, ** indicates the adequacy of this parameter, compared with the RL parameter).

When studying the level of accumulation of dry biomass in wheat germs, a significant decrease in the dry weight of the underground part of healthy wheat germs and infected with pathogenic fungus $F$. oxysporum was detected in the BL, compared with the RL and WL. There was also a significant increase in the dry weight content in the control versions of the RL (Figure 3). Such changes in the production of dry weight are most likely associated with the specificities of the morphological structure of wheat leaves, grown at different parts of the spectrum [Demina, 2015].

The activity of antioxidant enzymes of healthy and infested wheat germs

The key role in the emergence of hypersensitivity reaction and systemic stability under the influence of phytopathogens belongs to several signal molecules $\mathrm{H}_{2} \mathrm{O}_{2}$, NO, ethylacetat, SA and FA[Pieterse et al., 2009]. In case of infection, the synthesis and content of these compounds increase as in damaged, as in healthy tissues.
At the initial stage of pathogenesis, an oxidative explosion occurs in plant, at the site of the microorganism invasion, and then it leads to a hypersensitivity reaction [Chen, 2012]. In case of oxidative stress, enzyme antioxidants perform the protective function. The most important of them is catalase, which promotes the rapid utilization of hydrogen peroxide [Mhamdi, 2010].

Also, there are reasons to believe, that ascorbate peroxidase is involved in the formation of the crossresistance effect to several stressors [Browse, 2009; Przybyla, 2008]. However, in order to maintain its functions, it is necessary to have high level of ascorbate always.[Polleskaya, 2007].

Peroxidase is represented by proteins, which are produced by cells in response to the invasion of pathogen, and limit its spread by the formation of highly toxic media and the polymerization of oxycinnamic acids, during lignification of the plant cell walls [Sharma, 2012].

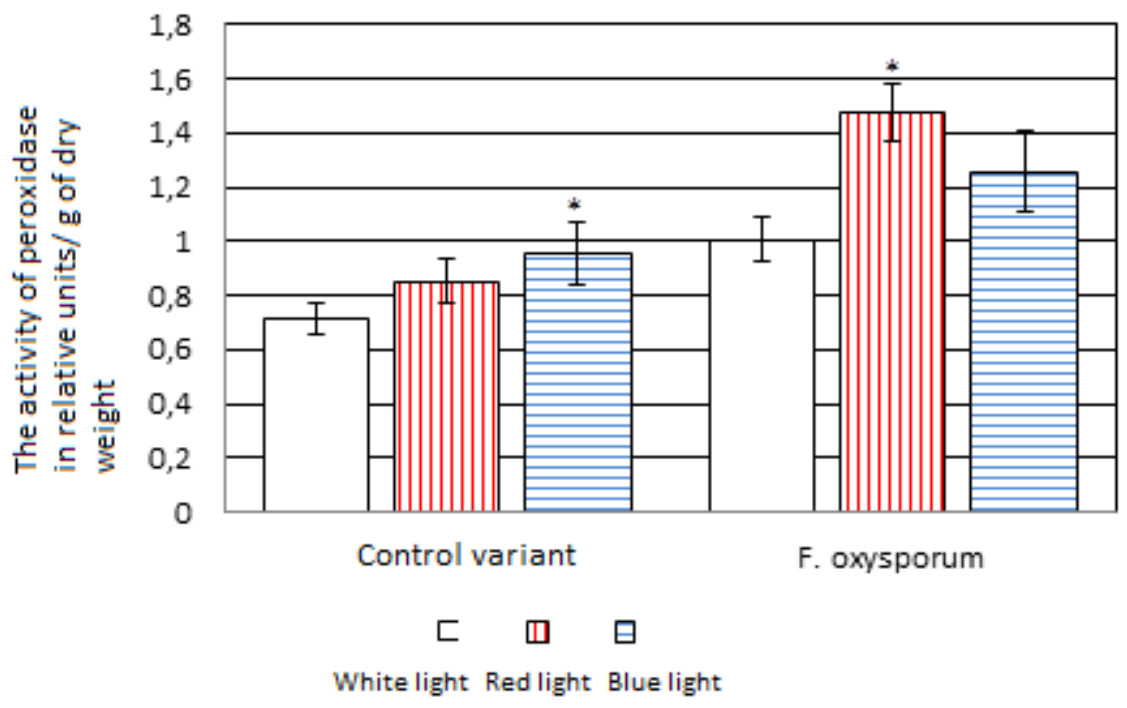

Figure 4-The influence of light of different spectral composition on the activity of soluble peroxidases in the wheat roots of the variety Kazan 560, in control variant and in case of their infestation with $F$. oxysporum (* indicates the adequacy of this parameter, compared with the WL parameter).

When studying the activity of peroxidase in wheat germs under the influence of light of different spectral composition, we found a significant increase in the samples, infected with phytopathogen, that indicated a high level of plant protection to penetration of antagonist (Figure 4). The highest activity of this enzyme was observed in the red light, in comparison with white light. Its activation under the influence of RL is realized through the activation of phytochromic system. Increased activity of peroxidase under the influence of RL in case of infection may indicate greater resistance of these sprouts to pathogens. Similar patterns were obtained by other authors. In experiments with the pre- 
exposure of separate leaves by RL, a direct activating effect of red light on the activity of peroxidase was observed [Kreslavskiy, 2012].
Wheat germs, which were not treated with the pathogen fungus, and were grown in the blue light, had the greatest oxidative status, which was manifested in the activation of peroxidases.
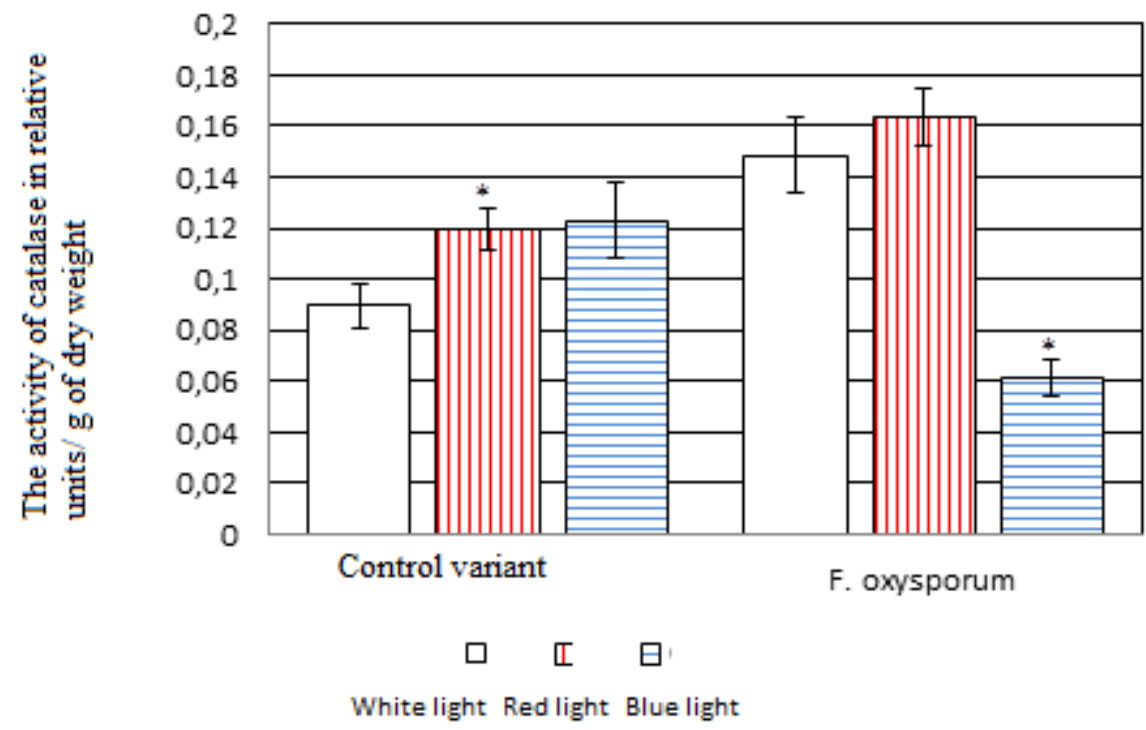

Figure 5 - The influence of light of different spectral composition on the activity of catalase in the wheat roots of the variety Kazan 560, in control variant and in case of their infestation with $F$. oxysporum (* indicates the adequacy of this parameter, compared with the WL parameter).

In the course of the research, we found, that under the influence of light of different spectral composition, in the roots of the test specimens (infected), grown in the RL and WL, a significant increase in the activity of catalase was observed, in contrast to wheat sprouts, grown in BL. It may indicate the insignificant role of this spectral region in the activation of this enzyme, in case of infection with F.oxysporum (Figure 5).
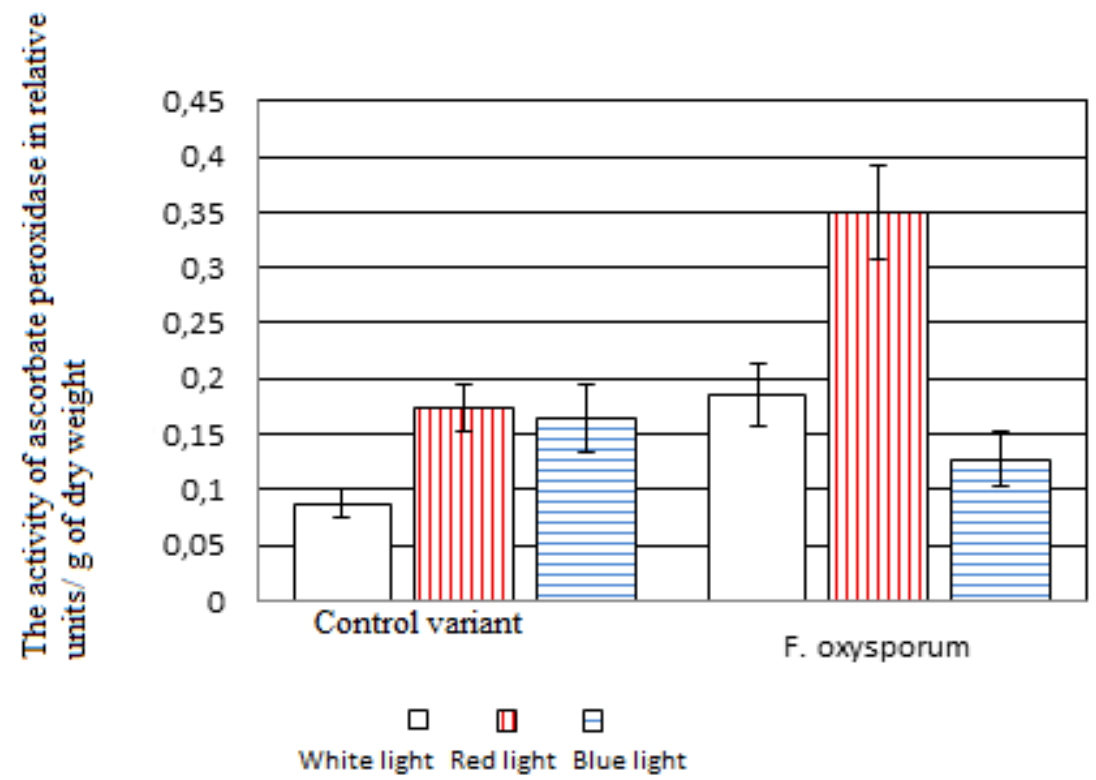

Figure 6 - The influence of light of different spectral composition on the activity of ascorbate peroxidase in the wheat roots of the variety Kazan 560, in control variant and in case of their infestation with F. oxysporum. 
The data, obtained in the course of the study, clearly show the increased content of ascorbate peroxidase in the infected wheat roots, under the influence of red light (Figure 6). A noticeable decrease in the activity of this enzyme is observed in the lighting of sprouts by BL. Under the influence of pathogen, the activity of ascorbate peroxidase decreases, which probably indicates the negative effect of this portion of spectrum on the penetration of $F$. oxysporum.
The MDA content in the roots of wheat germs It is known, that the toxic effect of pathogens on plants is manifested in the development of oxidative stress and the formation of reactive oxygen species (ROS). ROS are able to initiate lipid peroxidation, resulting in damage of membrane structures [Sharma, 2012]. It is believed, that such product of lipid peroxidation as malonic dialdehyde can be used as a biological indicator of the development of oxidative plant stress in pathogenesis [Shakirova, 2007].

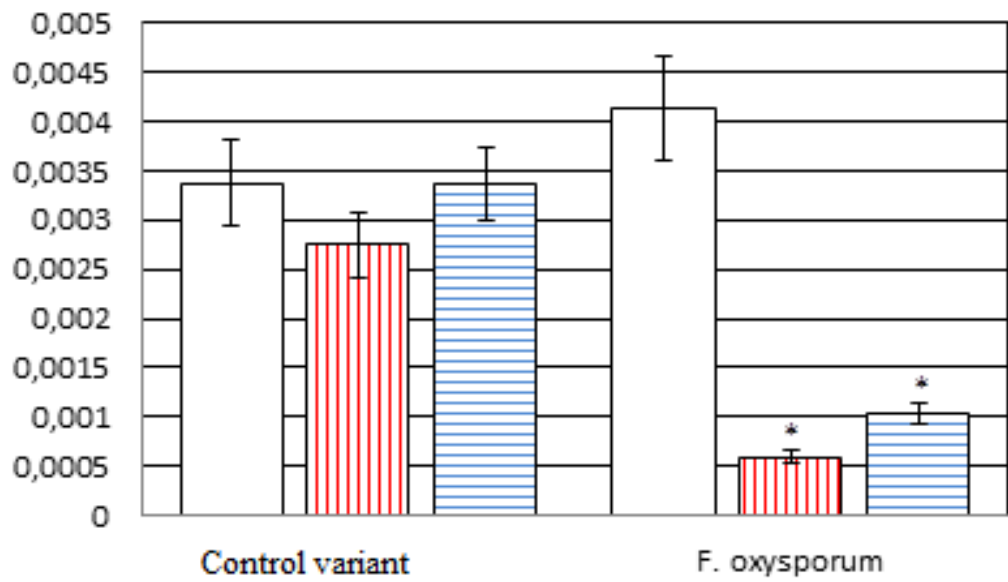

White light Red light Blue light

Figure 7 - The influence of light of different spectral composition on the content of malonic dialdehyde in wheat roots of the variety Kazan 560, in control variant and in case of their infestation with $F$. oxysporum (* indicates the adequacy of this parameter, compared with the WL parameter).

The study of the influence of light of different spectra on the MDA content in case of infection with pathogenic fungus showed, that wheat sprouts, grown in red light, had the least content of MDA. This indicates a high protective status, in case of phytopathogen invasion in the root system. Lignification of cell walls and the activity of antioxidant enzymes have a direct impact on the content of MDA. With a low activity of antioxidant enzymes in plants, grown in a blue light, the content of MDA in them is also low. This indicates the possible effect of other protective systems.

\section{Summary}

It was established, that the red spectral region stimulated the growth parameters of the aboveground and underground parts of wheat germs.

An increase in the production of dry weight by the underground part of wheat germs was found in case of infection with Fusarium oxysporum, to a greater extent in the red portion of the spectrum.

Red spectral region increased the activity of all studied antioxidant enzymes, under the influence of Fusarium oxysporum, that is one of the factors of their increased immunity. At the same time, only the 2390 peroxidase activity was increased in a blue light, and the activity of catalase and ascorbate peroxidase was decreased.

Colonization of the sprouts roots by Fusarium oxysporum reduces lipid peroxidation in red and blue light, that indicates their resistance to the studied phytopathogen.

\section{Conclusion}

As a result of the conducted studies, it was found, that the spectral composition of light, under the influence of phytopathogenic fungus on wheat germs, could be an important management tool for integrated programs of plant growth and development, and also could regulate the activity of the main antioxidant enzymes.

\section{Acknowledgements}

The work is performed according to the Russian Government Program of Competitive Growth of Kazan Federal University.

\section{References}

[1]Hammond-Kosack K.E., Jones J.D.G., Buchannan B., Gruissem W., Jones R. 2000.Response to plant Copyright @ 2018 Helix ISSN 2319 - 5592 (Online) 
pathogens.Biochemistry and molecular biology of plants. V. 1367: 1102 - 1157.

[2]Michielse C.B., Rep M. 2009. Pathogen profile update: Fusarium. Mol. Plant Pathogens.10: 311-324. [3]Leslie J.F., Bandyopadhyay R.,Mwangi M., Sylvester Aigbe O. 2006. Fusarium Species from the Cassava Root Rot Complex in West Africa.Phytopathological. 96: 673-676.

[4]Read A.F.2016. The evolution of virulence.Trends Microbiol. 2: 73 - 76.

[5]Casal J.J. 2013. Photoreceptor signaling networks in plant responses to shade.Annu. Rev. Plant Biol. 64: $403-427$.

[6]Xinyu Zhang, Juling Huai, Fangfang Shang, Gang $\mathrm{Xu}$, Weijiang Tang, Yanjun Jing, Rongcheng Lin.2017. A PIF1/PIF3-HY5-BBX23 Transcription Factor Cascade Affects Photomorphogenesis. Plant Physiol 174 (4): 2487-2500.

[7]Cheng M., Chory C. Fankhauser. 2004. Light signal transduction in higher plants. M. Annu. Rev. Genet. 38: 87 - 117.

[8]Ricci A., Dramis L., Shan R., Gartner W., Losi A. 2015.Visualizing the relevance of bacterial blue- and red-light receptors during plant-pathogen interaction. Environ.Microbiol. Rep.7(5):795-802.

[9]Schuerger A.C., Brown C.S.1997. Spectral quality affects disease development of three pathogens on hydroponically grown plants. Hort.Science. 32 (1):96-100.

[10] Ermakov A.I. 1987. Methods of biochemical research of plants. SPb: Kolos. Leningr.depart. 430p.

[11]Aebi H. 1984. Catalase in vitr.Methods Enzymol.105:121 - 126.

[12]Verma S. and Dubey R. S. 2003. Lead toxicity induces lipid peroxidation and alters the activities of antioxidant enzymes in growing rice plants. Plant Sci. 164: $645-655$.

[13]Kumar G.N., Knowles N.R. 1993. Involvement of auxin in the loss of apical dominance and plant growth potential accompanying aging of potato seed tubers. Can J. Bot.71:541-550.

[14]Alabouvette C., Olivain C., Migheli Q., Steinberg C. 2009. Microbiological control of soilborne phytopathogenic fungi with special emphasis on wilt-inducing Fusarium oxysporum.New Phytologist. 184: 529-544.

[15]Tsukagoshi H., Busch W., Benfey P.N. 2010. Transcriptional regulation of ROS controls transition from proliferation to differentiation in the root. Cell.143: 606-616.
[16]Yakushenkova T.P., Grakhantseva L.Sh.,Yunusov D.Yu. The red light increases content of cryoprotectant effect substances. 2012. 9th International Conference Plant Functioning Under Environmental Stress. Cracow. Poland. Pp. 80 - 81.

[17]Demina G.V. and Yakushenkova T.P. 2015. Leaf Anatomical Structure in Arabidopsis thaliana (L.)Heynh.Mutants Deficient in Photoreceptor. Research Journal of Pharmaceutical, Biological and Chemical Sciences. 6 (4): 141-147.

[18]Pieterse M.J., Antonio Leon-Reyes, Sjoerd Van der Ent, C.M. SaskiaVan Wees.2009. Networking by small-molecule hormones in plant immunity. Nature Chemical Biology. 5: $308-316$.

[19]Chen H. J., Wu S. D., Huang G.J., Shen C.Y., Afiyanti M. 2012. Expression of a cloned sweet potato catalase SPCAT1 alleviates ethephonmediated leaf senescence and $\mathrm{H}_{2} \mathrm{O}_{2}$ elevation. J. Plant Physiol. 169: 86-97.

[20]Mhamdi A., Queval G., Chaouch S.,Vanderauwera S., Van-Breusegem F. 2010. Catalase function in plants: a focus on Arabidopsis thaliana mutants as stress-mimic models. J. Exp. Bot. 61: 4197-4220.

[21]Browse J. 2009. Jasmonate passes muster: a receptor and targets for the defense hormone. Annu. Rev. Plant Biol. 60:183-205.

[22]Przybyla D., Gobel C., Imboden A., Hamberg M., Feussner I., Apel K. 2008. Enzymatic, but not non-enzymatic, 1O2-mediated peroxidation of polyunsaturated fatty acids forms part of the executer 1-dependent stress response program in the flu mutant of Arabidopsis thaliana.J. Plant. 54:236-248.

[23]Polleskaya O. 2007. Plant cell and reactive oxygen species.Moskova.KDU.

[24]Sharma P., Jha A.B.,Dubey R.S., Pessarakli M. 2012. Reactive oxygen species, oxidative damage, and antioxidative defense mechanism in plants under stressful conditions.Journal of Botany.- 1: 26.

[25]Kreslavskiy V.D. 2012. Preradiation individual spinach leaves with red light increases the resistance to UV radiation. Russian Journal of Plant Physiology.59 (6): 723-729.

[26]Shakirova F.M., Gimalov F.R., Aval'baev A.M., Sakhabutdinova A. R., Yuldashev R. A.2007. Structure of the TADHN gene for dehydrin-like protein of soft wheat and activation of its expression by $\mathrm{ABA}$ and 24-epibrassinolide.Russian Journal of Plant Physiology.-54: 115-120. 